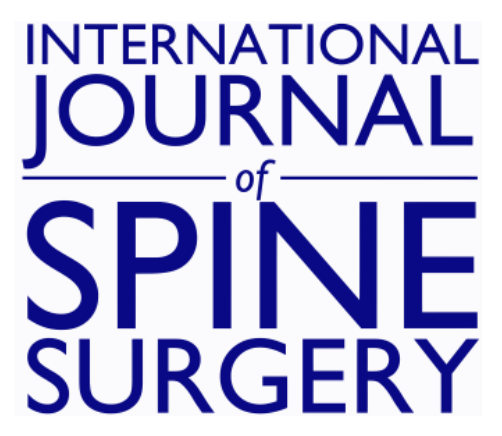

\title{
Successful Introduction of Full-Endoscopic Lumbar Interlaminar Discectomy in Sweden
}

JOEL BECK, OLOF WESTIN, MIKAEL KLINGENSTIERNA and ADAD BARANTO

Int J Spine Surg 2020, 14 (4) 563-570

doi: https://doi.org/10.14444/7075

http://ijssurgery.com/content/14/4/563

This information is current as of April 26, 2023.

Email Alerts Receive free email-alerts when new articles cite this article. Sign up at:

http://ijssurgery.com/alerts 


\title{
Successful Introduction of Full-Endoscopic Lumbar Interlaminar Discectomy in Sweden
}

\author{
JOEL BECK, MD, OLOF WESTIN, MD, PHD, MIKAEL KLINGENSTIERNA, MD, ADAD BARANTO, MD, \\ PHD \\ Department of Orthopaedics, Institute of Clinical Sciences at Sahlgrenska Academy, University of Gothenburg, and Sahlgrenska University Hospital, Gothenburg, \\ Sweden
}

\begin{abstract}
Background: The introduction of full-endoscopic lumbar discectomy (FELD) procedures has made it possible to challenge microscopic discectomy as the gold standard method to treat lumbar disc herniations.

Purpose: The aim of the present study is to investigate the introductory-phase postoperative clinical improvement for FELD patients regarding leg pain, patient-reported outcome measurements (PROMs), complications, reoperations, and learning curve analysis.

Methods: All patients who underwent FELD at Sahlgrenska University Hospital, Sweden, were prospectively included during 2013-2017. A total of 92 patients were enrolled and followed up for 1 year. The characteristics of the study population, degree of leg pain, complications, learning curve, and PROMs were retrieved from patient records and the National Quality Register for Spine Surgery (Swespine).

Results: The postoperative results demonstrated major improvements; leg pain measured by a numerical rating scale $(0-10)$ decreased from $7.4 \pm 2.25$ to $2.76 \pm 2.70$, with a mean improvement of $-4.54,(-3.62-5.46) 95 \%$ confidence interval (CI). The Oswestry Disability Index decreased by $30.48(-36.27-23.73)$ with a $95 \%$ CI, and the EuroQol-5D increased by $0.39(0.21-0.57) 95 \%$ CI. An assessment of the final surgical result showed that $91.6 \%$ ranked their general situation as better or much better. Specifically, regarding postoperative leg pain, 87\% regarded their leg pain as completely gone, much better, or somewhat better, while $13 \%$ regarded their leg pain as unchanged or worse. A learning curve analysis showed that for every 10th FELD procedure performed; the duration of surgery decreased by 2 minutes.

Conclusions: In our study, the introduction of FELD as a safe, quick procedure for the treatment of lumbar disc herniations can yield significant gains in patient-reported outcome measurements and pain reduction. The rate of recurrence and complications is comparable to that of standard surgery.
\end{abstract}

Endoscopic Minimally Invasive Surgery

Keywords: lumbar disc herniation, full-endoscopic lumbar discectomy (FELD), patient-reported outcome measurements (PROMs)

\section{BACKGROUND}

Disc herniation surgery has historically been performed using an extensive approach and laminectomy to remove the disc obtrusion from the spinal canal. ${ }^{1}$ Since the introduction of the current modern standardized disc herniation surgery in 1938 by Love et $\mathrm{al}^{2}{ }^{2}$ there has been a movement toward further minimization of tissue trauma. With the advent of microscopic procedures ${ }^{3}$ and percutaneous treatments, ${ }^{4}$ the surgery has evolved from a muscle-detaching procedure involving bony resection to truly minimally invasive techniques. Although these recent advances in surgical approaches and technologies likely cause less tissue trauma and provide a better surgical visualization, there is not as yet any conclusive evidence supporting the hypoth- esis that full-endoscopic lumbar discectomy (FELD) procedures for lumbar disc herniations are superior to the original open method.

On the contrary, some authors have found that endoscopically performed discectomies involve a higher rate of neurapraxia ${ }^{5}$ and a higher rate of recurrences when compared with standard surgery. ${ }^{6}$ Several of the most cited studies of FELD procedures in the lumbar spine have been written by the original inventors of the instruments and developers of the procedure, which could be considered a potential bias. ${ }^{4,-9}$ The introduction of FELD at our center was to be able to offer a less invasive way of treating lumbar disc herniations with decreased postoperative pain, potentially leading to a faster recovery. 
The purpose of the present study is to investigate postoperative outcomes for patients having FELD surgery. Data on leg pain, back pain, complications, learning curve, reoperations, patient-reported outcome measurements (PROMs), and the perceived impact of leg pain on quality of life were collected from electronic medical charts and the National Quality Register for Spine Surgery (Swespine).

\section{METHODS}

\section{Study Population}

All consecutive patients presenting with a disc herniation at the L4-L5 or L5-S1 levels and having a FELD procedure at the Spine Unit, Department of Orthopaedics, Sahlgrenska University Hospital, Gothenburg, Sweden, from 2013 to 2017 were enrolled.

\section{Inclusion and Exclusion Criteria}

The inclusion criterion for a FELD procedure to be performed was a herniated disc, verified by magnetic resonance imaging, completely contained within the spinal canal and with corresponding neurological findings. The exclusion criteria were cauda equina syndrome, previous lumbar surgery, and a congenital narrow spinal canal or severe spinal stenosis.

\section{Surgical Indication}

The indication for discectomy in this FELD series was unsustainable unilateral leg pain for more than 6 weeks, with or without motor and sensory deficit, that failed to respond to nonsurgical treatment.

\section{Surgical Technique}

All surgeries were performed at a major hospital. The authors acknowledge the possibility of a local anesthetic procedure, but due to local traditions and circumstances, a general anesthesia approach was utilized. Several patients were offered a local anesthetic approach but declined. The specific procedure has been described thoroughly in several previous articles. ${ }^{10,11}$ In brief, under general anesthesia with endotracheal intubation, the patient was positioned prone on a radiolucent table equipped with a Wilson frame. A single dose of antibiotics (Cloxacillin 2 g, Meda AB, Solna, Sweden) was administered. Utilizing radiological imaging, a $<1$ $\mathrm{cm}$ stab incision was made, and a dilator was inserted to the level of the medial border of the zygapophyseal joints at the disc herniation level. A beveled working sleeve and the endoscopic system (Vertebris, Richard Wolf GmbH, Knittlingen, Germany) was inserted, and a small resection of the ligamentum flavum was performed. The beveled sleeve and instrument were advanced through the ligamentum flavum and rotated to protect the neural structures medially. During constant lowpressure irrigation, the removal of the sequester/disc herniation was performed using micro rongeurs and scissors. Skin closure was performed using 1 absorbable suture (Vicryl, Ethicon Inc, Johnson and Johnson, Solna, Sweden). No drains were applied.

\section{Postoperative Care}

Following surgery, all the patients were allowed and encouraged to immediately mobilize without restriction. All the patients were discharged within 24 hours of the procedure. Physiotherapeutic instructions were provided for all patients.

\section{Follow-Up}

A follow-up wound check was scheduled at 2 weeks. All patients were advised to adopt an earlyreturn-to-work approach. The 1-year postoperative evaluation was performed using a mailed questionnaire form according to standard Swespine practice.

\section{Swespine and Data Collection}

The Swespine register is a national quality register that has been in use for more than 25 years. $^{12}$ It collects data from almost all units in Sweden performing spinal surgery. The register was used to retrieve PROMs, including data on leg pain rated by a numerical rating scale (NRS 0-10). Qualitative questions regarding perceived effect and outcome of surgery and a global assessment of outcome are also included.

\section{Statistics}

All the data are presented as means for normally distributed data and with median values when not normally distributed with the corresponding interquartile range (25th-75th percentile). The paired sample $t$ test with $95 \%$ confidence intervals was used. Statistical analyses were performed using SPSS version 20.0 (SPSS Inc, Chicago, Illinois) and JMP, version 15 (SAS Institute Inc, Cary, 
North Carolina). Student $t$ tests were conducted to confirm intergroup differences in cases with normal distribution. Mann-Whitney $U$ tests were used to compare variables between 2 groups with nonnormal distributions. For the categorical variables, $\chi^{2}$ tests and Fisher exact tests were performed between 2 independent groups. All $P$ values less than .05 were considered statistically significant.

\section{RESULTS}

A total of 92 patients, mean age 31.2 years (range 15-59), were enrolled and followed up in the study after 1 year. As displayed in Table 1, the median duration of preoperative pain was 9 months (range 0-24+). Seventy-six percent of the patients had preoperative positive straight leg raise test in the sciatica-affected extremity, and $34 \%$ had a motor affection in either the L5 or the S1 myotome. Sensory affection following the distribution pattern of either L5 or S1 was experienced by $53.7 \%$ of the patients. Twenty $(22 \%)$ patients had both motor and sensory disturbances preoperatively. A relatively young age distribution (mean age 31.22 , range 15-59) and a normal weight (body mass index 25.5) reflected our inclusion criteria.

\section{Patient-Reported Outcome Measurements}

In Table 2, the postoperative values at 12 months are presented. Leg pain (NRS), back pain (NRS), Oswestry Disability Index (ODI), EuroQol-5D (EQ$5 \mathrm{D})$, and the use of pain medication all showed significant improvements. At the 1-year follow-up, $87.0 \%$ of the study patients rated their leg pain symptoms as pain free, much better, or better. The improvements in leg and back pain $(-4.54$ and -2.46 , respectively) are clinically significant, ${ }^{13}$ and the overall perception of surgical result was rated as being satisfied or undecided (not worse) in $91.6 \%$ of cases after 1 year. In analogy with the changes in the leg and back pain scales, quality of life improved significantly on both the EQ-5D (0.39) and the ODI $(-30.48)$. The use of pain medication also decreased significantly, and $54 \%$ stated postoperatively that no medication at all was necessary, while a further $37 \%$ used it only occasionally as opposed to a $50 \%$ rate of opioid usage/dependency preoperatively.

\section{Surgical Outcomes}

The early cases in our FELD group represent the learning curve, causing prolonged surgical dura-
Table 1. Patient demographics and disc herniation data.

\begin{tabular}{lc}
\hline Parameter & Value (Mean and Range) \\
\hline Age, mean (range) & $31.22(15-59)$ \\
Gender (female) \% (n) & $50(46)$ \\
Body mass index, normal (range) & $25.5(20-33)$ \\
Preoperative pain duration, \% (n) & $n=92$ \\
$\quad<3$ mo & $15(14)$ \\
$3-12$ mo & $60(55)$ \\
$12-24$ mo & $12(11)$ \\
$>24$ mo & $13(12)$ \\
Disc herniation level, \% (n) & $1.1(1)$ \\
$\quad$ L4-S1 & $27.2(25)$ \\
L4-L5 & $71.7(66)$ \\
L5-S1 & \\
Laterality, \% (n) & $57.6(53)$ \\
$\quad$ Left & $42.3(39)$ \\
Right & $n=70,75.7(53)$ \\
Positive straight leg raise test, \% (n) & $n=76,53.7(40)$ \\
Sensory affection, \% (n) & $\mathrm{n}=76,34.2(26)$ \\
Motor affection, \% (n) &
\end{tabular}

tions. Surgical duration for the FELD procedure was recorded at a mean of $55 \pm 19$ minutes. The effect of the learning curve on surgical time is illustrated in the Figure. Average blood loss was $<7$ $\mathrm{mL}$. X-ray exposure was a mean of a 260-KAP (kerma area product) value. The average hospital stay was 0.8 days (range $0.5-2$ ), meaning that $67 \%$ of the patients had the procedure as day surgery with no overnight stay.

\section{Peri- and Postoperative Complications}

In Table 3 the operative data for the study group is illustrated. Three patients required conversion to open surgery perioperatively, $2(2 \%)$ patients pending technical difficulties (breakage of the camera, no soft interlaminar space), and $1(1 \%)$ patient because of a dural tear. In 1 case, this was due to osseous overgrowth limiting the visualization of the surgical field and in 2 cases due to equipment problems. There were no infections requiring surgical intervention, but 1 patient had a postoperative superficial infection requiring a short course of antibiotic treatment.

\section{Neuralgia and Nerve Injuries}

Preoperatively, $54 \%$ of the patients described changes in unilateral sensation in a dermatome consistent with nerve root impingement. Fifty percent $(n=46)$ of the patients reported that they had experienced transient changes or affected sensation postoperatively in a dermatome at follow-up. One of the patients in the study group sustained a laceration injury to the right S1 nerve root that was caused by microscissors. At revision, 
Table 2. Patient-reported outcome measurements.

\begin{tabular}{|c|c|c|c|}
\hline Parameter & Preoperative & Postoperative & $P$-Value \\
\hline EQ-5D & & & $<.001$ \\
\hline Mean (SD) & $0.31( \pm 0.32)$ & $0.70( \pm 0.28)$ & \\
\hline Mean difference $(95 \% \mathrm{CI})$ & & $0.39(0.21-0.57)$ & \\
\hline Median (IQR) & $0.16(0.09-0.69)$ & $50.00(28.50-58.00)$ & \\
\hline EQ visual analog scale & & & $<.001$ \\
\hline Mean (SD) & $44.26( \pm 18.02)$ & \multirow[b]{2}{*}{$29.62(22.63-36.60)$} & \\
\hline Mean difference $(95 \% \mathrm{CI})$ & & & \\
\hline Median (IQR) & $40.00(27.00-58.75)$ & $75.00(68.18-77.30)$ & \\
\hline ODI & & & $<.001$ \\
\hline Mean (SD) & $46.76( \pm 19.60)$ & $16.28( \pm 16.22)$ & \\
\hline Mean difference $(95 \% \mathrm{CI})$ & & $-30.48(-36.27-23.73)$ & \\
\hline Median (IQR) & $50.00(28.50-58.00)$ & $10(4.00-26.00)$ & \\
\hline Leg pain (NRS) & & & $<.001$ \\
\hline Mean (SD) & $7.4( \pm 2.25)$ & $2.76( \pm 2.70)$ & \\
\hline Mean difference $(95 \% \mathrm{CI})$ & & $-4.54(-3.62-5.46)$ & \\
\hline Median (IQR) & $8.0(7.0-9.0)$ & $2.0(0.25-4.5)$ & \\
\hline Back pain (NRS) & & & $<.001$ \\
\hline Mean (SD) & $5.3( \pm 2.79)$ & \multirow[b]{2}{*}{$-2.46(-3.29-1.64)$} & \\
\hline Mean difference $(95 \% \mathrm{CI})$ & & & \\
\hline Median (IQR) & $6.0(3.0-7.0)$ & $2.0(0.0-4.75)$ & \\
\hline Pain medication usage, $\%$ & $\mathrm{n}=48$ & $\mathrm{n}=62$ & $<.001$ \\
\hline None & 2 & 54.80 & \\
\hline Occasionally & 31 & 37.10 & \\
\hline Regularly & 67 & 8.10 & \\
\hline Opioid use & 50 & 22.90 & \\
\hline Return to work, $\%$ & $\mathrm{n}=38$ & $\mathrm{n}=62$ & $<.001$ \\
\hline Unemployed/student & 35.10 & 6.50 & \\
\hline Full-time & 2.70 & 69.40 & \\
\hline Part-time & 2.70 & 14.50 & \\
\hline Not able to work & 59.50 & 9.70 & \\
\hline
\end{tabular}

Abbreviations: EQ-5D, EuroQol 5D; CI, confidence interval; IQR, interquartile range; ODI, Oswestry Disability Index; NRS, numerical rating scale.

the nerve root was resutured with an acceptable outcome for the patient.

\section{Dural Tear}

One $(1 \%)$ case of dural tear requiring conversion to open repair was recorded. The patient was allowed to mobilize freely and did not have a prolonged rehabilitation or any adverse events relating to the surgery.

\section{Recurrence and Revision}

A recurrence of disc herniation, defined as a painfree interval of at least 6 weeks, with new onset of previous symptoms at the same disc level requiring revision surgery, occurred in 8 cases $(8.7 \%)$ within 1 year. One $(1 \%)$ patient required an early (within 1 week) revision due to the inadequate removal of disc material. Six revisions were performed using a miniopen surgical technique, and 2 revisions were performed using FELD.

\section{Global Assessment}

The patients made a general assessment of the surgical result regarding their leg pain. The majority $(87 \%)$ rated their postoperative leg pain as better, much better, or pain free. Despite perhaps not achieving complete restitution of their sciatica, $78 \%$ of the patients rated their overall postoperative attitude as satisfied as illustrated in Table 4.

\section{DISCUSSION}

The main finding in our case series is that FELD is a safe, quick procedure to introduce, with excellent clinical results and PROMs. However, a cumbersome learning curve and transient neurapraxia can be expected when introducing this surgical technique to treat lumbar disc herniation. During the past 10 years, FELD has evolved to become a viable alternative to other visualized surgical techniques for discectomy. ${ }^{14-22}$ Theoretical$1 y$, there are several advantages that would facilitate safer, less invasive surgery. Modern endoscopes offer an incomparable overview, illumination, and visualization of the surgical field when compared with either microscopic enhancement or loupes. With minimal skin incisions and the gentle dilatation of a fairly small 7-mm tissue canal, the FELD procedure is likely to cause less harm to the back muscles and ligaments and potentially reduce the amount of postoperative pain and complications. 


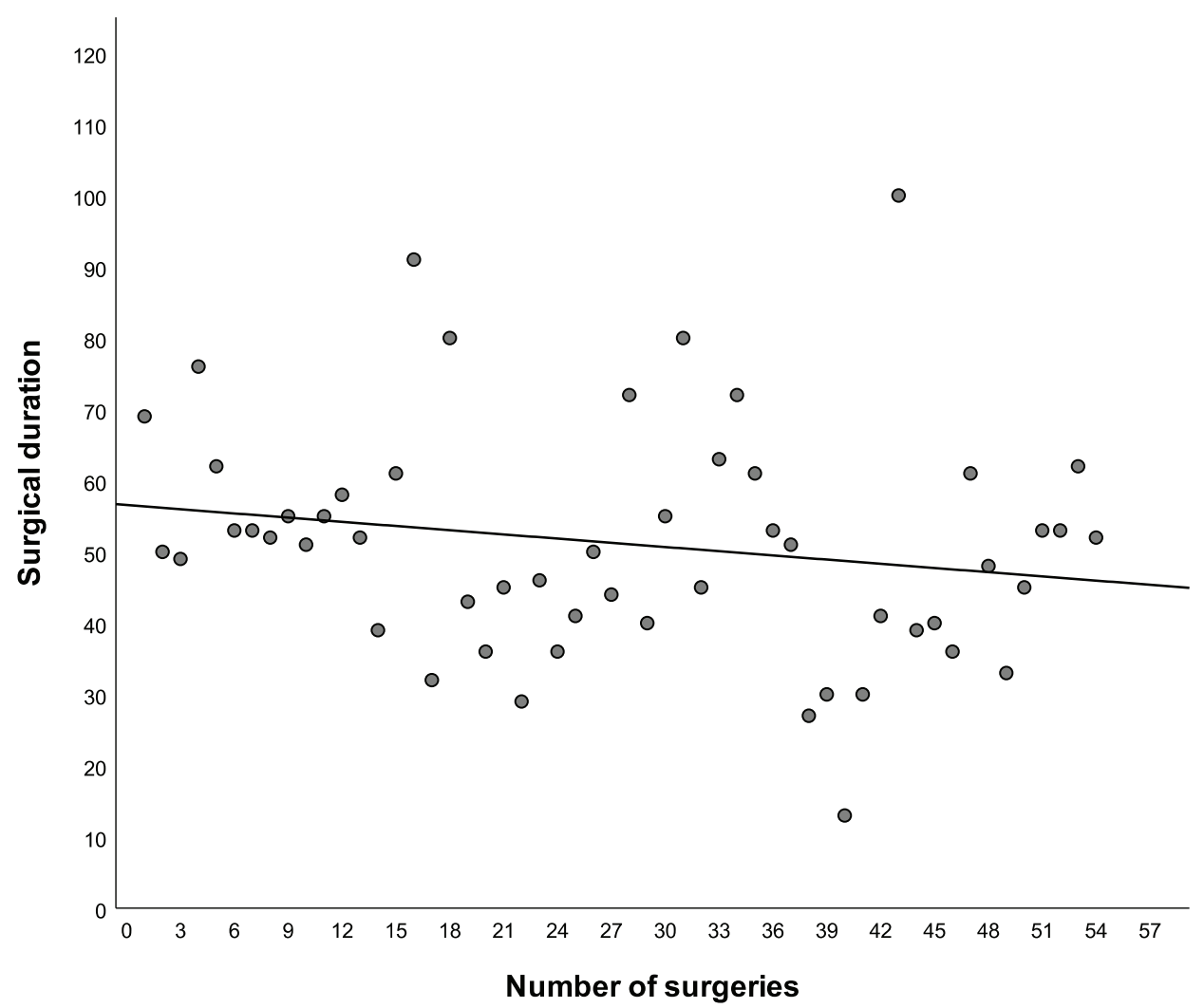

Figure. The effect of the learning curve on surgical duration.

\section{PROMs}

The improvements on the EQ-5D, EQ visual analog scale, and ODI were all significant $(P<.001)$ and well above the minimally clinical important difference. ${ }^{13}$ The quality control questions regarding the general assessment and leg pain-impacted quality of life reflect this. At the 1-year follow-up, $3(5 \%)$ patients rated their symptoms as worse than before surgery. As a comparison, standard lumbar discectomies carry a $5 \%$ corresponding failure rate according to the Swespine register.

\section{Hospital Length of Stay}

Regarding the length of stay in hospital, our results must be interpreted in the light of our attitude to free, unrestricted mobilization for our FELD patients. Most patients were able to leave to hospital the same day or the day after. Only 1 patient required a stay longer than 1 day. The reduced cost of a shorter hospital stay could potentially offset the high cost of initial investment in surgical equipment. From an international perspective, the average length of stay in Sweden is short, and a further decrease is highly unlikely.

\section{Dural Tear}

In this study, 1 patient had a visible dural tear $(1.1 \%)$ that was converted to open surgery. This is in line with previous studies that report a very low rate of dural tears in FELD procedures when compared to open lumbar disc herniation surgery. ${ }^{23,24}$ Due to the constant water irrigation and water pressure during the procedure, a dural tear may go unnoticed, and there is the risk of a delayed diagnosis. In our case series, we did not encounter any postoperative postural headache or late dural repairs. One reason for this may be that small dural tears are able to heal quickly in the relatively confined and undecompressed spinal canal following the FELD procedure.

Table 3. Surgical data.

\begin{tabular}{lcc}
\hline Parameter & n & Value, Mean (Range) \\
\hline Surgical duration, mean (range), min & 87 & $53.37(13-108)$ \\
Total time in theater, mean (range), min & 83 & $187.8(115-300)$ \\
Radiation, mean (range), min & 43 & $0.5(0.1-4.0)$ \\
Radiation, mean (range), KAP value & 43 & $260.63(15-2055)$ \\
Blood loss, mean (range), mL & 64 & $6.78(0-50)$ \\
Dural tears, n (\%) & 92 & $1(1.1)$ \\
Nerve injuries, n (\%) & 92 & $1(1.1)$ \\
In-hospital stay, mean (range), d & 92 & $0.8(0-2)$ \\
\hline
\end{tabular}

Abbreviation: KAP, kerma area product. 
Table 4. Leg pain-specific patient-reported outcome measurements.

\begin{tabular}{lc}
\hline Question & Response, \% (n) \\
\hline How do you rate your postoperative leg pain? & $\mathrm{n}=65$ \\
Pain free & $15(10)$ \\
Much better & $54(35)$ \\
Better & $18(12)$ \\
Unchanged & $8(5)$ \\
Worse & $5(3)$ \\
What is your attitude toward your final & $\mathrm{n}=59$ \\
surgical results? & $78.0(46)$ \\
Satisfied & $13.6(8)$ \\
Undecided & $8.5(5)$ \\
Dissatisfied & \\
\hline
\end{tabular}

\section{Recurrence Rate}

Minimally invasive discectomies have been known to involve a higher rate of recurrences requiring revision surgery. ${ }^{6}$ These findings have also been confirmed in our study, where $8.1 \%$ had a recurrence of their disc herniation within 1 year in comparison to open/microscopic surgery, which is at $7 \%$ in the Swespine register. One reason for this could be the very noninvasive nature of the procedure itself. Whereas an open surgery procedure would decompress all neurological structures inherent to the removal of ligamentum flavum, the FELD procedure is less a posterior decompression and more a minimal removal of a bulging disc or sequesterectomy. We speculate that our revision rate reflects the minimally decompressive properties of this procedure.

\section{Nerve Injuries}

The majority of the patients suffered from preoperative sensory deficits $(53.7 \%)$ or motor deficits $(34.2 \%)$. Eighteen patients had both motor and sensory deficits. Nine cases $(9 \%)$ of postoperative permanent sensory nerve loss or severe dysesthesia were documented in the patients' records. All these patients also had severe symptoms before surgery. In this study, transient postoperative neurapraxia was noted in a high proportion of the patients at discharge but not at the follow-up visits. Despite our patients' self-reported experiences of postoperative neurapraxia, the vast majority expressed satisfaction with the procedure, and only 3 $(5 \%)$ rated their general assessment of the procedure as unchanged or worse pain. We speculate that the high rate of neurapraxia reflects our learning curve but is perhaps also to some extent inherent to the procedure. The diameter of the beveled working sleeve might be considered overly intrusive in a narrower-than-normal spinal canal. The disc herniation and accompanying inflammation are also key risk factors in creating a hypersensitive environment for the nerve root. While being a truly minimal invasive method, the procedure has the potential to cause excessive nerve injuries if handled improperly within the spinal canal. As a result of this, a proper patient selection is crucial, and in many instances, the insertion of the working sleeve below the ligamentum flavum is perhaps not merited.

\section{Infections}

One significant advantage of the FELD procedure could be the extremely low rate of deep wound infections ( $0 \%$ in our series). One superficial wound infection was treated uneventfully using oral antibiotics.

\section{Limitations}

Previous studies have pointed to a troublesome learning curve. ${ }^{25,26}$ It has been suggested that the first 50 cases are an appropriate learning period for the surgical team. ${ }^{7}$ In this single-center study, starting with the FELD technique, the data contain a significant number of early-learning-phase cases that could potentially skew the data.

The preoperative duration of leg pain could be regarded as long from an international perspective (median 9 months, range $0-24$ ), but this is in accordance with Swedish practice and is comparable to other types of disc herniation procedure within the Swedish spine register. A prolonged time to surgery may carry the risk of the patients suffering from more severe neurological preoperative deficits, and this may perhaps affect the final outcome with less restitution of preoperative symptoms.

All consecutive patients were recruited from a single center. All the surgeries were performed by a limited number of surgeons with varying degrees of experience with the described method during the introductory phase at our center. Another limitation is the dropout rate at the 1-year follow-up. All nonresponders received repeated reminders. However, two previous analyses of loss to follow-up in European spine registries have shown only minor negative effects on the statistical validity. ${ }^{27-29}$

\section{CONCLUSIONS}

In our study, the introduction in Scandinavia of FELD as a safe, quick procedure for the treatment of lumbar disc herniations has yielded significant gains in patient-reported outcome measurements 
and leg pain. The rate of recurrence and complications is comparable to that of standard surgery.

\section{ACKNOWLEDGMENTS}

The authors acknowledge the financial support of the Medical Society of Gothenburg, Sweden; grants from the Swedish state under the agreement between the Swedish government and the county councils; the ALF agreement (ID no. 238801); the Orthopedic Spine Research Foundation; and the Spine Unit at Sahlgrenska University Hospital. The authors would also like to thank medical statistician Christer Johansson, OrigoVerus AB, Gothenburg, Sweden, for statistical assistance.

\section{REFERENCES}

1. Mixter WJ, Ayer JB, Barr JS. The intervertebral disk. $\mathrm{Br}$ Med J. 1940;1(4141):829-830.

2. Love J, Walsh MN. Protruded intervertebral disks: report of one hundred cases in which operation was performed. JAMA. 1938;111(5):396-400.

3. Williams RW. Microlumbar discectomy: a conservative surgical approach to the virgin herniated lumbar disc. Spine. 1978;3(2):175-182.

4. Kambin P, Brager MD. Percutaneous posterolateral discectomy. Anatomy and mechanism. Clin Orthop Relat Res. 1987(223):145-54.

5. Xie TH, Zeng JC, Li ZH, et al. Complications of lumbar disc herniation following full-endoscopic interlaminar lumbar discectomy: a large, single-center, retrospective study. Pain Phys. 2017;20(3):E379-E387.

6. Feng $\mathrm{F}, \mathrm{Xu} \mathrm{Q}$, Yan $\mathrm{F}$, et al. Comparison of 7 surgical interventions for lumbar disc herniation: a network metaanalysis. Pain Phys. 2017;20(6):E863-E871.

7. Ruetten S, Komp M, Merk H, Godolias G. Fullendoscopic interlaminar and transforaminal lumbar discectomy versus conventional microsurgical technique: a prospective, randomized, controlled study. Spine. 2008;33(9):931-939.

8. Ruetten S, Komp M, Merk H, Godolias G. Recurrent lumbar disc herniation after conventional discectomy: a prospective, randomized study comparing full-endoscopic interlaminar and transforaminal versus microsurgical revision. J Spinal Disord Tech. 2009;22(2):122-129.

9. Yeung AT. The evolution of percutaneous spinal endoscopy and discectomy: state of the art. Mt Sinai Journal Med. 2000;67(4):327-332.

10. Ruetten S, Meyer O, Godolias G. Endoscopic surgery of the lumbar epidural space (epiduroscopy): results of therapeutic intervention in 93 patients. Minim Invasive Neurosurg. 2003;46(1):1-4.

11. Ruetten S, Komp M, Merk H, Godolias G. Use of newly developed instruments and endoscopes: full-endoscopic resection of lumbar disc herniations via the interlaminar and lateral transforaminal approach. J Neurosurg Spine. 2007;6(6):521530 .

12. Stromqvist B, Fritzell P, Hagg O, Jonsson B, Sanden B,
Swedish Society of Spinal Surgeons. Swespine: the Swedish spine register: the 2012 report. Eur Spine J. 2013;22(4):953-974.

13. Werner DAT, Grotle M, Gulati S, et al. Can a successful outcome after surgery for lumbar disc herniation be defined by the Oswestry Disability Index raw score? Glob Spine J. 2019; 10(1):2192568219851480.

14. Nakamura JI, Yoshihara K. Initial clinical outcomes of percutaneous full-endoscopic lumbar discectomy using an interlaminar approach at the L4-L5. Pain Phys. 2017;20(4):E507-E512.

15. Phan K, Xu J, Schultz K, et al. Full-endoscopic versus micro-endoscopic and open discectomy: a systematic review and meta-analysis of outcomes and complications. Clin Neurol Neurosurg. 2017;154:1-12.

16. Cong L, Zhu Y, Tu G. A meta-analysis of endoscopic discectomy versus open discectomy for symptomatic lumbar disk herniation. Eur Spine J. 2016;25(1):134-143.

17. Hussein M. Minimal incision, multifidus-sparing microendoscopic diskectomy versus conventional microdiskectomy for highly migrated intracanal lumbar disk herniations. $\mathrm{J} \mathrm{Am}$ Acad Orthop Surg. 2016;24(11):805-813.

18. Ruan W, Feng F, Liu Z, Xie J, Cai L, Ping A. Comparison of percutaneous endoscopic lumbar discectomy versus open lumbar microdiscectomy for lumbar disc herniation: a meta-analysis. Int JSurg. 2016;31:86-92.

19. Li XC, Zhong CF, Deng GB, Liang RW, Huang CM. Full-endoscopic procedures versus traditional discectomy surgery for discectomy: a systematic review and meta-analysis of current global clinical trials. Pain Phys. 2016;19(3):103-118.

20. Choi KC, Kim JS, Park CK. Percutaneous endoscopic lumbar discectomy as an alternative to open lumbar microdiscectomy for large lumbar disc herniation. Pain Phys. 2016;19(2):E291-E300.

21. Rasouli MR, Rahimi-Movaghar V, Shokraneh F, Moradi-Lakeh M, Chou R. Minimally invasive discectomy versus microdiscectomy/open discectomy for symptomatic lumbar disc herniation. Cochrane Database Systematic Rev. 2014(9):Cd010328.

22. Kambin P, Savitz MH. Arthroscopic microdiscectomy: an alternative to open disc surgery. Mt Sinai $J$ Med. 2000;67(4):283-7.

23. Ahn Y, Lee HY, Lee SH, Lee JH. Dural tears in percutaneous endoscopic lumbar discectomy. Eur Spine J. 2011;20(1):58-64.

24. Chen X, Chamoli U, Lapkin S, Castillo JV, Diwan AD. Complication rates of different discectomy techniques for the treatment of lumbar disc herniation: a network meta-analysis. Eur Spine J. 2019;28(11):2588-2601.

25. Lee DY, Lee SH. Learning curve for percutaneous endoscopic lumbar discectomy. Neurol Medico-Chir. 2008;48(9):383-388; discussion 8-9.

26. Ao S, Wu J, Tang Y, Zhang C, Li J, Zheng W, et al. Percutaneous endoscopic lumbar discectomy assisted by $\mathrm{O}$ arm-based navigation improves the learning curve. BioMed Res Int. 2019;2019:6509409.

27. Endler P, Ekman P, Hellstrom F, Moller H, Gerdhem P. Minor effect of loss to follow-up on outcome interpretation in the Swedish spine register. Eur Spine J. 2020;29(2):213-220.

28. Hojmark K, Stottrup C, Carreon L, Andersen MO. Patient-reported outcome measures unbiased by loss of follow- 
up. Single-center study based on DaneSpine, the Danish spine surgery register. Eur Spine J. 2016;25(1):282-6.

29. Solberg TK, Sørlie A, Sjaavik K, Nygaard ØP, Ingebrigtsen $\mathrm{T}$. Would loss to follow-up bias the outcome evaluation of patients operated for degenerative disorders of the lumbar spine? Acta Orthop. 2011;82(1):56-63.

Disclosures and COI: Ethical permission was given by the Regional Ethical Review Board in Gothenburg at the Sahlgrenska Academy, Gothenburg University, Gothenburg, Sweden (ID no. 75317). All the patients in the study gave their informed consent to the medical procedures, data handling, and publication of data. All patient data have been anonymized. The study was approved by the local institutional review board. All the participants agreed to the scientific publication of data. All the authors agreed to the publication of the article. The data sets used and analyzed during the current study are available from the corresponding author in response to a reasonable request. None of the authors has any competing interest or conflict of interest to declare. This work was supported by grants from the Swedish state under the agreement between the Swedish government and the county councils, the ALF agreement (ID no. 238801).

Corresponding Author: Joel Beck, MD, Department of Orthopaedics, Sahlgrenska University Hospital/Sahlgrenska, SE-413 45 Göteborg, Sweden. Phone: +46 31 3421000; Email: pjm. beck@gmail.com.

Published 28 August 2020

This manuscript is generously published free of charge by ISASS, the International Society for the Advancement of Spine Surgery. Copyright (c) 2020 ISASS. To see more or order reprints or permissions, see http://ijssurgery.com. 